\title{
ASTRAL : un logiciel pour l'évaluation des conséquences d'un rejet accidentel de radionucléides dans l'environnement
}

\author{
H. MAUBERT*, P. RENAUD*, J.-C. BERNIÉ*, \\ G. DE DROUAS**, P. FACHE*, J.-M. MÉTIVIER* \\ (Reçu le 19 mars 1997, révisé le 6 mai 1997, accepté le 9 mai 1997)
}

RÉSUMÉ ASTRAL est acronyme d'، assistance technique en radioprotection postaccidentelle ». Si un rejet important de radionucléides dans l'environnement se produisait, il faudrait rapidement évaluer la concentration des radionucléides dans les milieux et les produits alimentaires, en déduire l'exposition potentielle aux rayonnements des populations concernées, prévoir l'évolution de la situation et proposer différents scénarios de gestion des zones contaminées. A ces fins, il a été décidé il y trois ans environ de créer un logiciel utilisable par un ensemble relativement large d'agents gréant les centres de crise, réalisant des études prévisionnelles, ou s'occupant habituellement du contrôle de l'impact des rejets de routine. Le point de départ des estimations est le dépôt sur le sol des radionucléides. La phase de dispersion atmosphérique et les conséquences de l'exposition au nuage et aux radionucléides à vie courte ne sont pas traitées ici. Les calculs effectués concernent à la fois l'évolution dans le temps des concentrations des radionucléides dans les sols et les éléments de la chaîne alimentaire, ainsi que l'évaluation des doses efficaces dues à l'exposition externe et interne. Ces grandeurs sont comparées aux limites et niveaux d'intervention en vigueur. Différentes simulations de la gestion des zones contaminées peuvent être conduites par application de contre-mesures. Ainsi, le logiciel ASTRAL peut être utilisé comme un élément d'aide à la décision.

ABSTRACT ASTRAL : a software for the estimation of accidental release of radionuclides in the environment.

ASTRAL is an acronym for "radioprotection in post accidental situation". Should an important release of radionuclides in the environment occur, it would be necessary to rapidly assess the concentration of radionuclides in the environment and in the elements of the food chain, derive from that the potential exposure of concerned populations to the radiations, forecast the evolution in time of this exposure and propose different scenarios of remediation actions in the contaminated zones. For this purpose it was decided three years ago to create a software fit for a relatively large group of personnel involved in the management of radioactive releases, or staffing crisis centres. The starting point of the evaluations is the ground deposition of radionuclides. The atmospheric dispersion phase, the exposure to the plume and to short-lived radionuclides is not treated here. The calculations performed concern the evolution in time of concentrations in the soil and the elements of the food-chain, and the evaluation of the doses due to external and internal exposure. The results are compared to the admissible limits and intervention levels. Different simulations of management of contaminated zones, by implementation of countermeasures may be conducted. So, the ASTRAL software may be used as an element for decision making.

Institut de protection et de sûreté nucléaire, Département de protection de l'environnement, CE Cadarache, 13108 Saint Paul-lez-Durance, Cedex

** Institut de protection et de sûreté nucléaire, Département de prévention et d'Étude des accidents, B.P. 6, 92265 Fontenay-aux-Roses, Cedex. 


\section{Introduction}

Cet article a pour objet de présenter de façon synthétique les principales fonctionnalités du logiciel ASTRAL « assistance technique en radioprotection post-accidentelle ». Ce logiciel calcule les principales grandeurs permettant d'évaluer les conséquences d'un dépôt de radionucléides sur le sol. Typiquement, une session de calcul se déroule de la façon suivante : sélection des données d'entrée, calcul des concentrations des radionucléides dans les sols et les produits de la chaîne alimentaire, calcul des doses. Ces premiers calculs sont faits en considérant qu'aucune contre-mesure n'est appliquée.

D'autres cas de calcul peuvent être lancés en considérant l'application de contre-mesures, ce qui permet de quantifier le gain que I'on en attend. Le développement informatique ${ }^{(1)}$ du logiciel a été réalisé selon un plan d'assurance qualité particulier, ce qui garantit la traçabilité des programmes et leur maintenabilité. Sur le plan scientifique, qui inclut les modèles mathématiques et leurs paramètres, la procédure d'assurance qualité des documents scientifiques et techniques de l'IPSN a été suivie. Électricité de France a cofinancé le logiciel ASTRAL, a participé à sa définition en exprimant ses besoins propres et en effectuant des essais sur les maquettes successives du produit.

\section{Description du logiciel}

\subsection{Organisation des calculs et architecture générale}

Le logiciel est conçu pour utiliser des données spécifiques d'un site nucléaire situé sur le territoire français. À la suite d'un rejet de radionucléides dans l'atmosphère, la composition des dépôts sur le sol varie en fonction de la distance du point de rejet de l'installation accidentée, et de conditions atmosphériques particulières (précipitations en particulier). Il est alors possible de définir une série de «zones d'études », chaque zone étant constituée d'une collection d'entités géographiques (communes, régions agricoles, départements) sur lesquelles les dépôts sont considérés comme identiques et homogènes (zones iso-dépôt).

Un exemple de zones d'études est représenté sur la figure 1, tirée d'un exercice de crise appelé «Becquerel » traitant d'un accident hypothétique sur le site nucléaire de Saclay, et qui s'est déroulé en octobre 1996.

(1) Le développement informatique a été assuré par la société GFI Informatique, Direction régionale MidiMéditerranée, Parc club du golf, Bâtiment 20, BP 207000, 13796 Aix-en-Provence Cedex 3. 


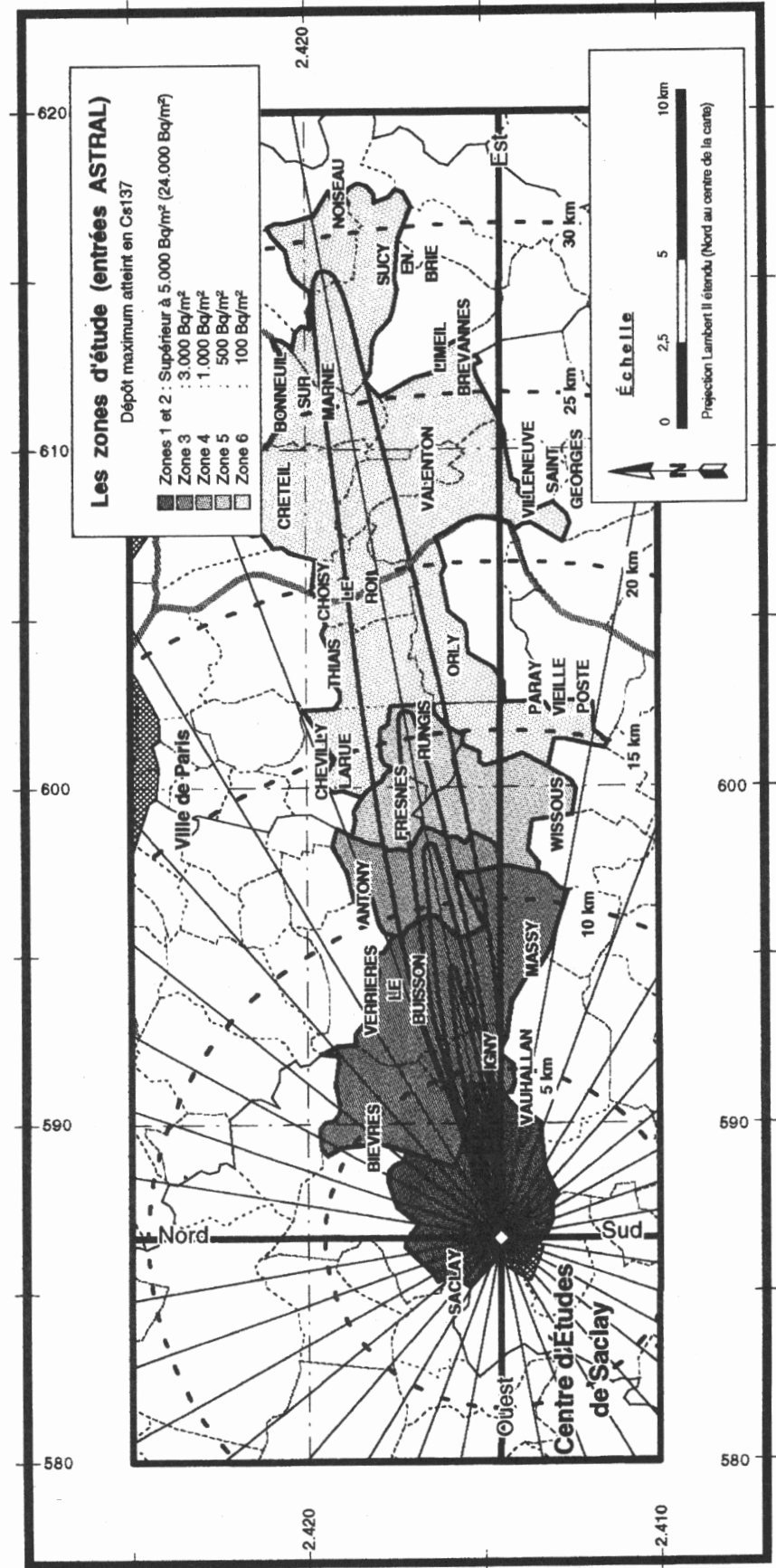

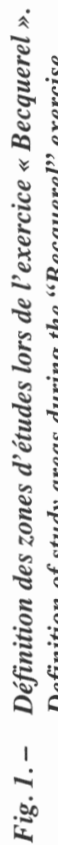


Pour chaque zone d'étude, les données spécifiques de l'environnement du site nucléaire sont extraites de bases de données, puis les calculs sont réalisés. Il s'agit du « cas d'étude » initial, qui, systématiquement, ne prend en compte aucune contre-mesure. L'opérateur peut ensuite étudier des variantes de l'étude initiale, en introduisant l'influence de contre-mesures, comme des actions sur les pratiques agricoles, des interdictions de consommation, etc. Il peut ainsi générer plusieurs cas d'études (cas initial +2 ), comparer les résultats entre eux et proposer une stratégie de gestion des territoires contaminés. Les mêmes opérations peuvent être reproduites sur d'autres zones iso-dépôt relatives à un même accident. L'ensemble des études constitue alors un « classeur» qui peut, par exemple, correspondre à l'ensemble des évaluations faites lors d'un exercice de crise particulier. Une fois les calculs réalisés, les résultats sont renvoyés au niveau de la base de données pour exploitation et représentation. Sur le plan informatique cela induit une architecture dite «client-serveur».

\subsection{Brève description des bases de données}

\subsubsection{Données concernant l'agriculture}

Sur le plan agricole, l'administration française commercialise un certain nombre de fichiers statistiques. Pour ASTRAL nous en avons surtout exploité deux : le fichier «AGRISTAT »qui fournit des données selon un découpage départemental et le fichier «ARISTIDE» qui fournit des données selon le découpage des régions agricoles ${ }^{(2)}$ et des communes. Certaines des données sont réactualisées tous les ans. Les fonds statistiques sont souvent assez volumineux (on compte environ 36500 communes en France) et parfois peu exploitables à cause de la protection de certaines d'entre elles pour des raisons dites de « secret statistique ». De plus il y a environ 4000 entrées dans la nomenclature des produits agricoles «AGRISTAT». Pour ASTRAL, il a donc été nécessaire de procéder à des regroupements ou agrégations en :

- 14 catégories de produits végétaux (légumes feuilles, légumes fruits, céréales, fruits, fourrages) et de 14 produits dérivés d'origine végétale (conserves, surgelés, extraits, concentrés) ; les données sont relatives aux superficies, et si disponibles, aux productions annuelles.

- 14 catégories de produits d'origine animale (viande de bœuf, de mouton, de volaille, de porc, lait...) et de 14 produits dérivés d'origine animale (conserves, produits laitiers) ; les données sont relatives aux nombres de têtes et si disponibles, aux productions annuelles.

(2) Les régions agricoles regroupent des communes sur la base de caractéristiques agricoles similaires, et non sur une appartenance administrative forcément arbitraire. Il y a en France près de 400 régions agricoles 
Par catégorie de produits végétaux, des calendriers agricoles des productions ont été recueillis, et différenciés selon 4 aires géographiques en France. Il en a été de même pour les calendriers d'affouragement des animaux.

\subsubsection{Données concernant la population}

Un fichier des populations a été acheté à l'INSEE (recensement de population 1990). Il contient, pour toutes les communes françaises, le nombre d'habitants sans double compte, par classes d'âges de 1 an et jusqu'à 100 ans. Ce fichier est réactualisé lors de chaque recensement. L'avantage de disposer de classes d'âge aussi fines que 1 an est de pouvoir les regrouper «à façon ». Ainsi, pour les besoins de la radioprotection, ces données ont été agrégées en 6 classes d'âges telles qu'elles sont définies dans la directive 96/29/Euratom du 13 Mai 1996: (0 - 1 an, $1-2$ ans, $2-7$ ans, $7-12$ ans, $12-17$ ans et $>17$ ans).

\subsubsection{Données radioécologiques}

Les radionucléides pris en compte actuellement dans ASTRAL sont les suivants : ${ }^{131} \mathrm{I},{ }^{137} \mathrm{Cs},{ }^{134} \mathrm{Cs},{ }^{106} \mathrm{Ru},{ }^{103} \mathrm{Ru},{ }^{90} \mathrm{Sr},{ }^{89} \mathrm{Sr}$. Cette liste peut éventuellement être étendue. Tous les paramètres, facteurs et coefficients mentionnés ci-après sont relatifs à ces radionucléides. Les facteurs relatifs aux transferts radioécologiques comme le sont les transferts foliaires, racinaires, plantes - animaux sont extraits soit des principales compilations de la littérature (Communautés européennes, 1996 ; IAEA, 1994 ; ICRP, 1991) soit de valeurs établies grâce aux expérimentations précédemment menées dans notre service.

Des coefficients relatifs à l'efficacité des contre-mesures ont été rassemblés. Les principales contre-mesures que l'on peut simuler sont l'enlèvement de la végétation, le décapage des sols, l'ajout d'engrais, l'ajout d'absorbants dans la ration alimentaire des animaux, le retrait du pâturage, et les interdictions de consommation.

Les données précédentes sont complétées dans la base par un recueil des données relatives aux transformations agro-alimentaires des produits, par exemple les transformations du lait en produits laitiers (beurre, fromages...).

\subsubsection{Données radiologiques}

Les coefficients de dose engagée pour les modes « ingestion » et « inhalation » sont extraits de la directive des Communautés européennes (1996). Les coefficients de dose par irradiation externe due au dépôt au sol sont issus des fichiers du Oak Ridge national laboratory. Dans cette section des bases de données figurent les paramètres des modes de vie des populations : temps de résidence à l'intérieur ou à l'extérieur des lieux d'habitation, facteurs de protection des habitations, débits respiratoires... 


\subsubsection{Limites de consommation et niveaux d'intervention}

En ce qui concerne les limites de commercialisation, les valeurs publiées au journal officiel des communautés européennes (1989) figurent dans les bases de données. D'autre jeux de valeurs peuvent y figurer également comme les limites de commercialisation édictées par la Communauté européenne après l'accident de Tchernobyl et toujours en vigueur, ou éventuellement d'autres jeux, comme les normes russes ou même des valeurs arbitraires. Figurent également dans les bases de données des limites annuelles d'incorporation (LAI) issues du rapport CIPR 61 (1991). Comme pour les limites de commercialisation, on pourrait envisager d'autres jeux de LAI.

\subsection{Calculs et résultats}

\subsection{Modèles mathématiques}

On entend ici par modèle un jeu d'équations associé à un jeu de paramètres. Pour ASTRAL, un modèle mathématique original nommé modèle-ASTRAL a été établi à partir d'une large analyse bibliographique et, partiellement, de résultats d'expérimentations. Ce modèle est dynamique, c'est-à-dire qu'il donne l'évolution des grandeurs calculées en fonction du temps. Deux modes d'utilisation sont prévus :

- le mode « crise », dans lequel les calculs se déroulent de façon pratiquement automatique une fois les caractéristiques de l'accident traité (dépôts, dates) définies; ce mode est typiquement destiné aux centres de crise,

- le mode « étude ", où l'utilisateur peut accéder à des fonctionnalités plus évoluées, comme des modifications de facteurs de transfert ; ce mode permet plus particulièrement des études prévisionnelles plus détaillées.

Les calculs sont menés sur une durée de trois ans après la date du dépôt, ce qui garantit la prise en compte de deux cycles agricoles complets au moins. Pour des durées plus longues, il est possible d'extrapoler les résultats obtenus la troisième année. Enfin le logiciel est limité à l'environnement terrestre, les eaux douces ou marines n'étant pour l'instant pas prises en compte.

\subsubsection{Calculs de concentration}

Ces calculs permettent d'obtenir l'évolution des concentrations en fonction du temps des différents radionucléides du dépôt dans les productions agricoles ainsi que dans les produits dérivés. Il peuvent être faits pour des radionucléides ou des groupes de radionucléides. Pour les productions continues telles que légumes feuilles, maraîchers, lait, viande, ces concentrations apparaissent sous forme graphique (Fig. 2). Les calendriers agricoles des récoltes et les calendriers d'affouragement des animaux sont automatiquement pris en compte. 


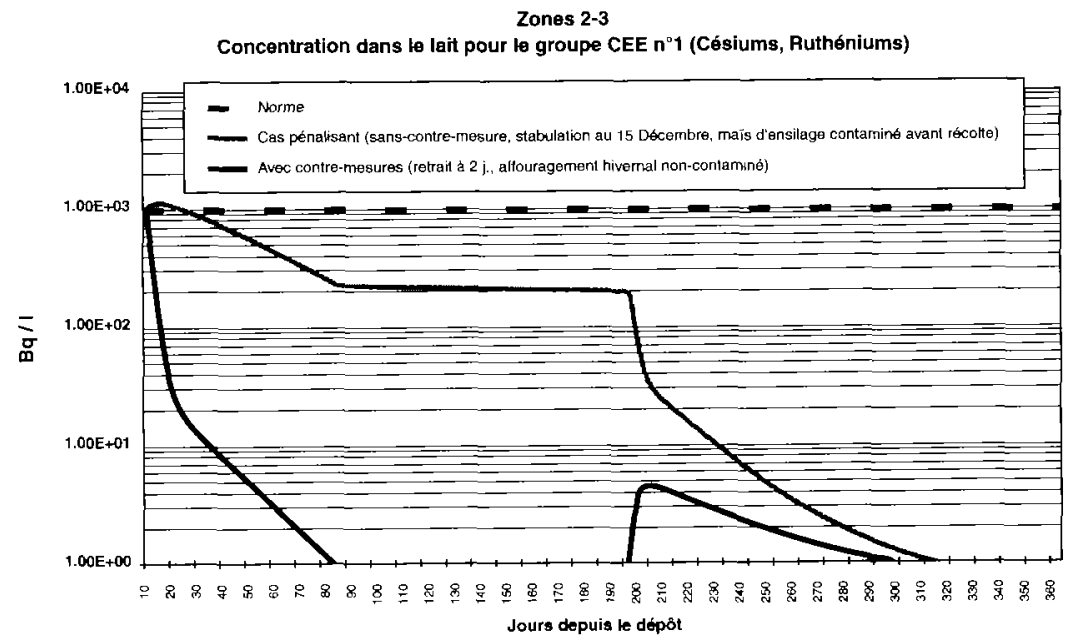

Fig. 2. - Exemple de courbes de concentrations des radionucléides dans le lait. Extrait de l'exercice « Becquerel».

Example of radionuclides concentration curves in milk. From the "Becquerel" exercise.

Sur le même graphique la limite de commercialisation retenue apparaît également de façon à repérer rapidement les périodes pendant lesquelles des problèmes peuvent se poser. Si dans certains cas d'études des contre-mesures ont été sélectionnées, les courbes intègrent l'influence de ces sélections et permettent d'évaluer le gain obtenu.

Dans le cas de productions discontinues telles que les céréales, des représentations graphiques ne s'imposent pas et des résultats tabulés sur les deux ou trois récoltes suivant l'accident peuvent être obtenus.

Au terme de cette étape de calculs il est possible de tracer des courbes d'incorporation de radionucléides par un individu de la population. (Fig. 3).

\subsubsection{Calculs d'indices de concentration et de quantités contaminées}

Plus que la valeur absolue, c'est la valeur relative de la concentration radioactive (par rapport à une norme de commercialisation) qui est parlante pour les décideurs. Aussi, pour la représentation cartographique des résultats, il a été décidé de calculer des indices de concentrations, définis comme les rapports des concentrations pour un produit donné à la limite de commercialisation retenue. Ces indices sont regroupés en classes, qui permettent, grâce à un code de couleur, de repérer les zones où les concentrations sont très inférieures, dans le voisinage, ou supérieures à la limite retenue. Une illustration de cette classification est représentée sur la figure 4. 
Zone 1, 1ère année : évolution de l'incorporation journalière de CS137 (sans contre-mesures) Cas vraisemblable : faible autarcie, maïs d'ensilage non contaminé, stabulation 15 Décembre (sommation des contributions des groupes de produits)

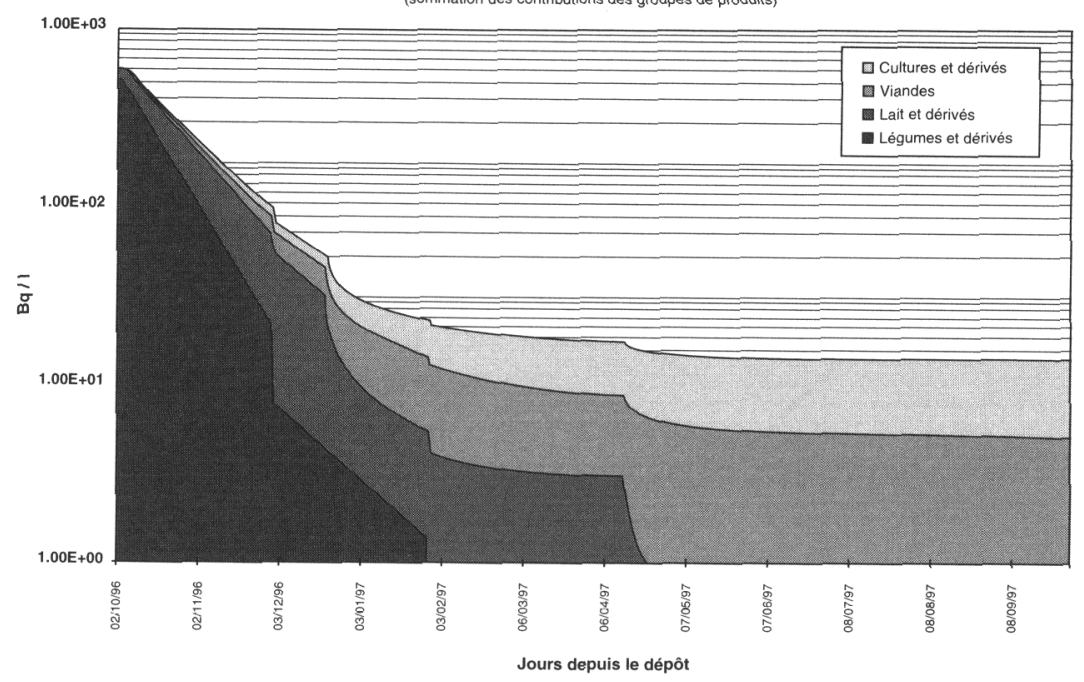

Fig. 3. - Incorporation de ${ }^{137} \mathrm{Cs}$ pendant un an après le dépôt. Exercice «Becquerel» Incorporation of ${ }^{137} \mathrm{Cs}$ during one year after deposition. "Becquerel" exercise.

Une opération de contre-mesure conduit généralement au stockage de produits agricoles contaminés, qui constitueront à terme des déchets et qu'il est, de ce fait, nécessaire de caractériser. Une évaluation des quantités de produits se situant dans une classe donnée d'indices de concentrations est donc réalisée. Les quantités sont extraites des bases de données «agriculture », lorsqu'elles sont disponibles. On peut par exemple savoir que dans telle ou telle zone, on attend $x$ tonnes de blé se situant 10 fois au-dessus de la limite de commercialisation.

\subsubsection{Calculs de doses}

Seules les doses efficaces sont calculées dans la version présente d'ASTRAL. Il est possible d'obtenir les évaluations de doses de façon très analytique (Fig. 3) :

- par période de durée ajustable après le dépôt ; par exemple de 0 à 30 jours, de 31 jours à 1 an et de 1 an à 3 ans,

- pour chaque période il est possible d'obtenir une ventilation par voie d'atteinte : irradiation externe, irradiation interne par inhalation due aux resuspensions, et ingestion, 


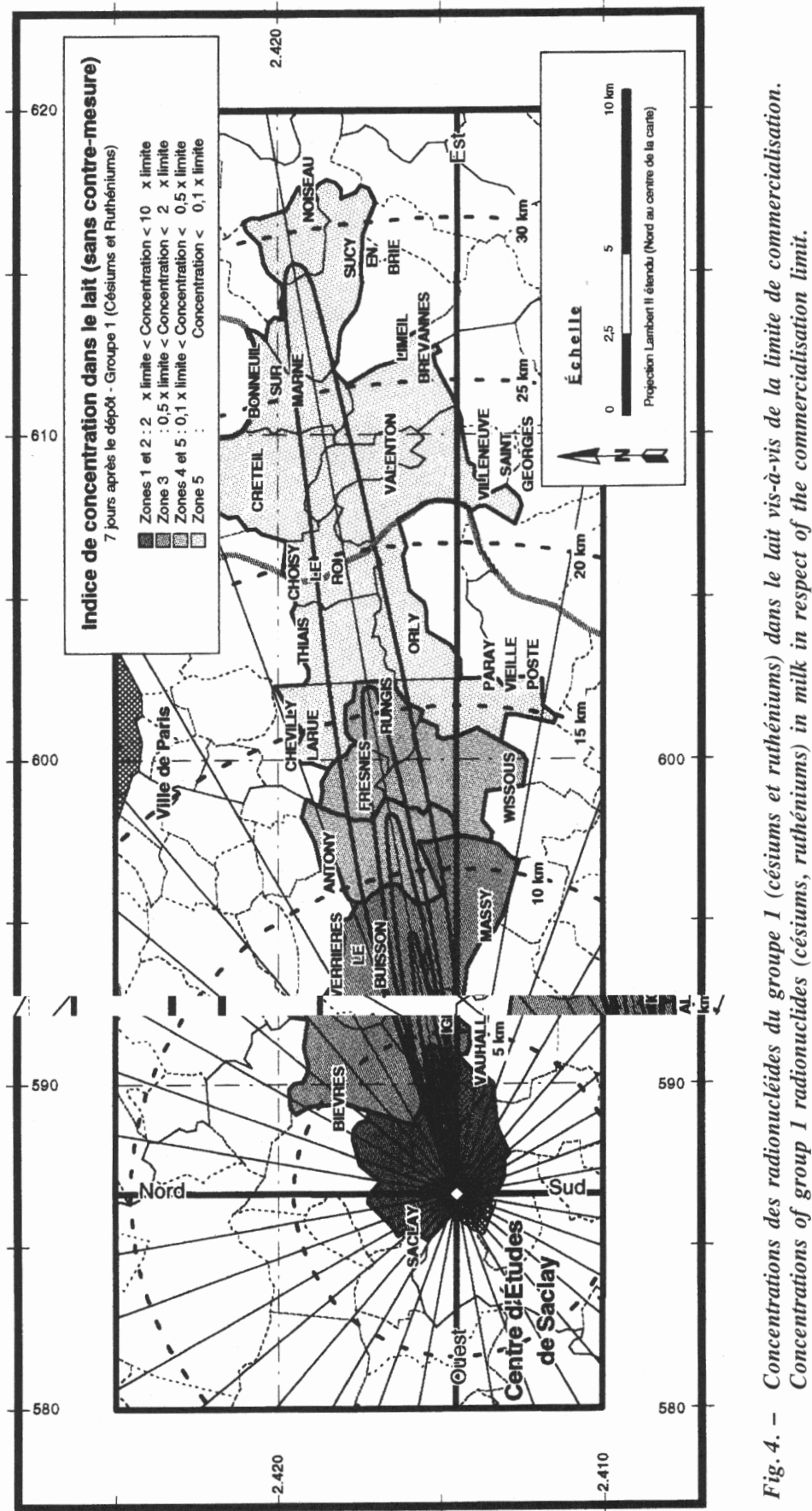


- pour la voie ingestion on peut obtenir une ventilation par produit et par radionucléide ou par groupe de radionucléides,

- du fait que, selon les activités professionnelles de la population, plusieurs types d'exposition aux radiations sont envisageables, en particulier au niveau du temps de séjour à l'extérieur des lieux d'habitation, tous les résultats précités peuvent être ventilés par mode de vie, ou par classe d'âge.

Total sans contre-mesure : $7 \mathrm{mSv}$

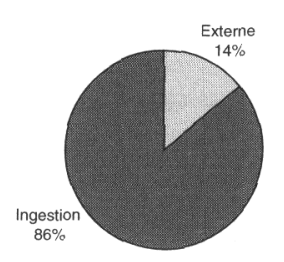

Contribution de la voie d'atteinte

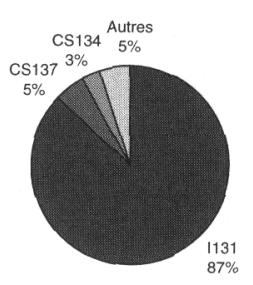

Contribution des radionucléides

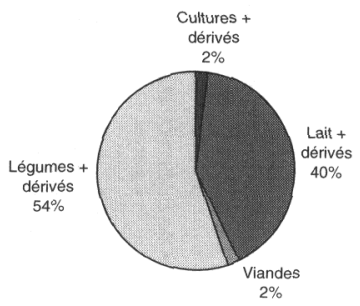

Contribution des produits

Fig. 5. - Exercice « Becquerel ». Représentation des doses efficaces pour la première année dans la zone 1 (Saclay, Vauhallan). Cas vraisemblable : faible autarcie.

"Becquerel" exercise. Representation of the effective doses for the first year in zone 1 (Vauhallan, Saclay). Likely case : weak auto-consumption.

Une estimation des doses collectives par classe d'âge est possible car les effectifs de population sont connus. Si pour une classe d'âge des modes de vie différents ont été choisis, 1'opérateur doit indiquer les proportions de la population affectés à chaque mode de vie.

Si entre deux cas d'études pour une même zone des contre-mesures ont été simulées, on peut obtenir un calcul des doses évitées. On entend par dose évitée la différence entre la dose calculée hors application de la contre-mesure et de la dose calculée après application de la contre-mesure. Il s'agit donc du gain attendu de l'action.

\subsection{Ergonomie du logiciel}

\subsubsection{Principes généraux}

L'ergonomie du logiciel a fait l'objet de soins particuliers. En effet, le volume de données à manipuler est très important, tant pour les données initiales que pour les résultats. Il fallait donc définir l'ergonomie du logiciel de façon que la manipulation de calculs complexes reste simple pour l'opérateur. Il a donc été fait appel à des objets graphiques tels qu'ascenseurs, onglets, zones de listes, auxquels 1'usage des logiciels commerciaux a habitué les utilisateurs de la 
micro-informatique. De plus, on a recherché la présentation des informations indispensables à l'écran qui soit la plus claire possible en séparant les champs que l'opérateur doit renseigner des informations utiles pour avoir connaissance du déroulement de la session de travail. À titre d'exemple on donne ci-après le cheminement pour obtenir les premiers calculs, et un écran de sélection des résultats de calculs de doses.

\subsubsection{Lancement de l'application}

En mode crise, seuls deux ou trois écrans sont nécessaires pour lancer les calculs. Il s'agit :

- d'initialiser le classeur, c'est-à-dire de lui donner un nom et de définir les données communes à l'ensemble des travaux qui seront faits dans ce cadre, les coordonnées du site, la date de l'accident, la proportion des radionucléides dans le dépôt-sol, les caractéristiques météorologiques qui prévalaient lors des dépôts, le choix des limites de commercialisation et des niveaux d'intervention,

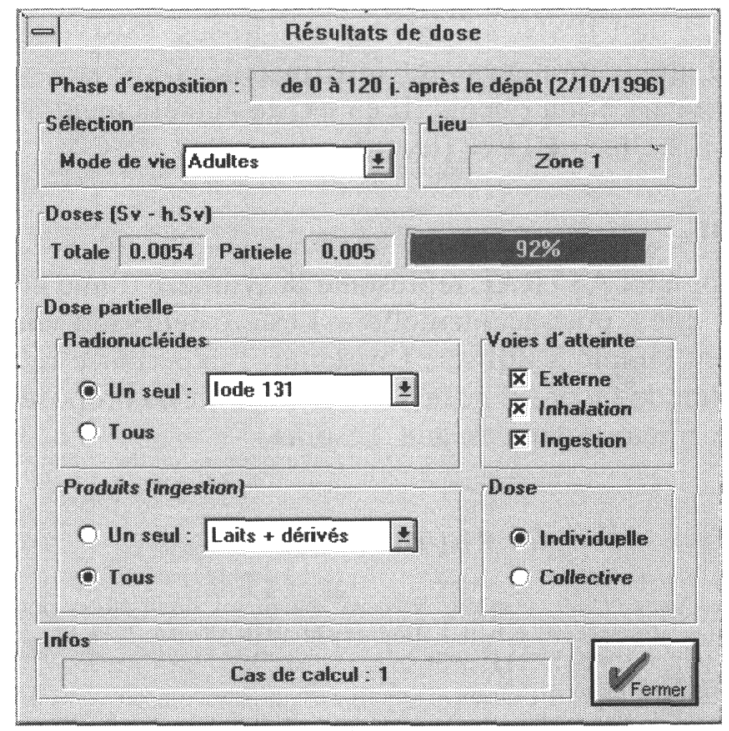

Fig. 6. - Copie d'un écran de sélection des résultats d'ASTRAL.

Copy of an ASTRAL results selection screen.

- d'initialiser les zones d'études, ou zones iso-dépôt, en définissant l'ensemble des entités géographiques qui les constituent (communes par exemple), ce qui permettra d'extraire les données agricoles et démographiques relatives à ces zones, 
et en affectant une valeur de dépôt à chacune d'elle. Il est à signaler que le processus d'extraction de ces informations d'étude pourra ultérieurement faire appel à un système d'informations géographiques.

L'écran de sélection des résultats de doses est un bon exemple de 1'ergonomie du logiciel. Les résultats de doses peuvent être ventilés de nombreuses façons différentes : par groupe de population (mode de vie), par période d'exposition, par voie d'atteinte, par produit, par radionucléide... tous les choix possibles sont représentés dans un écran unique, qui permet à la fois d'avoir une représentation globale des résultats et une analyse détaillée.

\section{Implantation et disponibilité}

Le logiciel ASTRAL fonctionne selon une architecture «client-serveur ». Il peut être implanté du côté client sous quatre systèmes d'exploitation informatiques différents : Windows 3.11, Windows 95, Windows NT $(3.51,4)$, Unix (HP et SUN), et du côté serveur sous trois systèmes d'exploitation informatiques différents: Windows 95, Windows NT $(3.51,4)$, Unix (SUN). La version de production est implantée sous Unix du côté client, et du côté serveur elle est destinée à prendre place parmi les logiciels déjà disponibles au centre technique de crise de l'IPSN, à Fontenay-aux-Roses. Des versions contenant des copies de l'application peuvent être installées sur des ordinateurs de bureau (PC), serveurs et/ou clients. Il en existe actuellement à I'IPSN/SERE (Cadarache) et à EDF/SEPTEN (Lyon).

\section{Conclusion}

La création du logiciel ASTRAL représente la synthèse d'une grande partie de la radioécologie dite "post-accidentelle ». Les créateurs ont voulu en faire un outil relativement simple à utiliser. Un retour d'expérience relatif à son utilisation est attendu de façon, si nécessaire, à pouvoir en proposer de nouvelles versions encore mieux adaptées aux besoins.

\section{RÉFÉRENCES}

Communautés européennes (1989) Conseil. Niveaux maximaux admissibles pour les denrées alimentaires et les aliments pour bétail. Règlement EURATOM 2218-89 du 18 juillet 1989, J.O.C.E., 22 Juillet 1989, L 211, 1-3.

Communautés européennes (1996) Conseil. Directive 96/29/Euratom du conseil, du 13 Mai 1996, fixant les normes de base realtives à la protection sanitaire de la population et des travailleurs contre les dangers résultant des rayonnements ionisants, J.O.C.E., 29 Juin 1996, L 159.

IAEA (1994) Handbook of parameter values for the prediction of radionuclides transfer in temperate environments. Tech. Rep. Ser. 364.

ICRP (1991) Annual Limits on Intake of Radionuclides by Workers, based on the 1990 recommendations, ICRP publication 61. Pergammon press.

Müller H., Pröhl G. (1993) ECOSYS-87 : a dynamic model for assessing radiological consequences of nuclear accidents. Health Phys., 64 (3), 232.

UIR (1987) Fifth report of the work group on soil to plant transfer factors. Union Internationale des radioécologistes, RIVM, The Netherlands. 